\title{
Energy transfer between Jahn-Teller systems in $\mathrm{RbMnF}_{3}$
}

\author{
F R Wünsch and W Gebhardt \\ Institute of Physics II, University of Regensburg, D-8400 Regensburg, Federal Republic \\ of Germany
}

Received 5 July 1988

\begin{abstract}
Energy transfer in $\mathrm{RbMnF}_{3}$ was studied by time-resolved spectroscopy of ${ }^{4} \mathrm{~T}_{1 \mathrm{~g}}$ exciton and trap luminescence. Uniaxial stress was used to lift the orientational degeneracy of the Jahn-Teller distorted $\mathrm{MnF}_{6}$ octahedra. Stress also reduces the excitonic transfer rates considerably. The most drastic reduction of transfer and a non-exponential decay was observed with [ $\left[\begin{array}{lll}1 & 0\end{array}\right]$ stress. Evidence for two-dimensional transfer under [ $\left[\begin{array}{llll}1 & 1 & 0\end{array}\right]$ stress is given by computer simulation of random-walk processes and by a calculation of nearestneighbour transfer integrals.
\end{abstract}

\section{Introduction}

Non-radiative energy transfer in organic and inorganic systems has been studied for many years. In the simplest case the excited state of the active ion or molecule is an orbital singlet and the ensemble of the interacting particles may be described by a twolevel system.

In the present work a system with threefold degenerate orbital $\mathrm{T}_{1 \mathrm{~g}}$ states of $\mathrm{Mn}^{2+}$ ions has been studied by time-resolved luminescence spectroscopy. The $T_{1 \mathrm{~g}}$ states of $\mathrm{Mn}^{2+}$ ions couple strongly with the lattice modes of local symmetry e. Therefore the quasi-particles which mediate the resonant energy transfer between $\mathrm{Mn}^{2+}$ ions are exciton polarons. The local Jahn-Teller effect of the type $\mathrm{T} \times \mathrm{e}$ lifts the threefold electronic degeneracy but adds an orientational degeneracy of distorted $\mathrm{MnF}_{6}$ octahedra. Uniaxial stress can be used to lift this remaining degeneracy. In the system under consideration the stress splitting is much larger than the dispersion of the ${ }^{4} \mathrm{~T}_{1 \mathrm{~g}}$ excitonic states; this dispersion is smaller than $1 \mathrm{~cm}^{-1}$. It is therefore possible to select an orbital singlet or doublet state for energy transfer experiments by application of stress. It will be shown that the transfer rates depend strongly on this selection and that in the case that the excited state is an orbital singlet the transfer is highly anisotropic and is confined to lattice planes.

In $\S 2$ of this paper we give a brief description of the electronic and vibronic properties of the $\mathrm{Mn}^{2+}$ system in $\mathrm{RbMnF}_{3}$. A model for incoherent energy transfer is considered in $\S 3$, together with computer simulations of the transfer process. In $\S 4$ we describe the experiments, and in $\S 5$ the results at temperatures between 1.4 and $20 \mathrm{~K}$ are given. In $\S 6$ we discuss the results using the calculated splitting of the ${ }^{4} \mathrm{~T}_{1 \mathrm{~g}}$ states and compare them with theoretical predictions and with computer simulations of the energy transfer. 
In $\S 7$ a microscopic model for transfer rates is discussed using the known orbital states. Section 8 finally gives a summary and conclusions.

\section{Electronic and vibronic structure}

$\mathrm{RbMnF}_{3}$ crystallises in an undistorted cubic perovskite structure at room temperature and does not exhibit a structural phase transition when it is cooled down to liquid helium temperature (figure 1). The $\mathrm{Mn}^{2+}$ ions form a simple cubic lattice with lattice constant $a=4.25 \AA$. Each $\mathrm{Mn}^{2+}$ ion is surrounded by six $\mathrm{F}^{-}$ions at a distance of $a / 2$ and by eight $\mathrm{Rb}^{+}$ions at a distance of $(1 / \sqrt{ } 2) a$. Below $83 \mathrm{~K} \mathrm{RbMnF}_{3}$ becomes an antiferromagnet. The point symmetry at the $\mathrm{Mn}^{2+}$ ion is $\mathrm{O}_{\mathrm{h}} \cdot \mathrm{Mn}^{2+}$ has a ${ }^{6} \mathrm{~A}_{1 \mathrm{~g}}$ ground state and a ${ }^{4} \mathrm{~T}_{1 \mathrm{~g}}$ first excited state which is the initial state of the luminescence. The phonon-free transition energy is

$$
E\left({ }^{4} \mathrm{~T}_{1 \mathrm{~g}}\right)-E\left({ }^{6} \mathrm{~A}_{1 \mathrm{~g}}\right)=18232 \mathrm{~cm}^{-1} .
$$

This transition is spin- and parity-forbidden, resulting in a relatively long radiative lifetime of about $60 \mathrm{~ms}$ (Strauss et al 1976).

The Hamiltonian of the system can be separated into three parts

$$
H=H_{\mathrm{E}}+H_{\mathrm{L}}+H_{\mathrm{E}, \mathrm{L}}
$$

where $H_{\mathrm{E}}$ denotes the electronic, $H_{\mathrm{L}}$ the lattice part and $H_{\mathrm{E}, \mathrm{L}}$ the electron-lattice interaction. All parts of the Hamiltonian can be written in terms of local coordinates because of the weak coupling between neighbouring manganese sites.

The electronic part $H_{\mathrm{E}}$ may be expressed using local creation and annihilation operators $c_{n i}^{+}$and $c_{n i}$

$$
H_{\mathrm{E}}=\varepsilon_{0} \sum_{n} \sum_{i=1}^{3} c_{n i}^{+} c_{n i}-\frac{1}{2} \sum_{n n^{\prime}}^{\prime} \sum_{i=1}^{3} J_{n n^{\prime}}^{i} c_{n i}^{+} c_{n^{\prime} i} .
$$

Here the index $i$ denotes one of the three ${ }^{4} \mathrm{~T}_{1 \mathrm{~g}}^{i}$ states of a manganese ion at lattice site $n$. The second term describes tunnelling between $\mathrm{Mn}^{2+}$ ions. Off-diagonal tunnelling, i.e. between states with different orbital components $i$, is neglected. The transfer integral $J^{i}$ must be identical by symmetry for all components $i$. Hence the index $i$ will be omitted. Transfer is only possible between ions of equal spin (see e.g. Pacheco 1983). Therefore transfer in the antiferromagnetic phase occurs on one magnetic face-centred cubic (FCC) sublattice with a nearest-neighbour distance of $a \vee 2=6.0 \AA$. We restrict the transfer

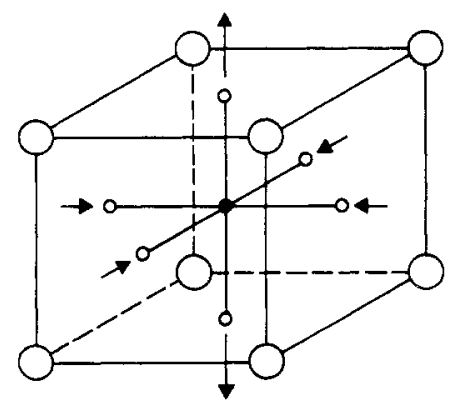

$$
\begin{aligned}
& -\mathrm{Mn}^{2+} \\
& 0 \mathrm{~F}^{-} \\
& \bigcirc \mathrm{Rb}^{+}
\end{aligned}
$$

Figure 1. Lattice cell of $\mathrm{RbMnF}_{3}$. The arrows indicate the eigenvector of the vibrational mode $Q_{\ominus}$ 
integral $J_{n n^{\prime}}$ to nearest neighbours only. In the special case of isotropic transfer we have

$$
J_{n n^{\prime}}= \begin{cases}J & \text { for }|R|:=\left|R_{n^{\prime}}-R_{n}\right|=6 \AA \\ 0 & \text { for all other distances. }\end{cases}
$$

Then, the energy of the electronic system can be described by a band of Frenkel excitons

$$
E(k)=E_{0}+2 J \cos (k \cdot R) .
$$

The remaining terms of equation (2.2), $H_{\mathrm{L}}$ and $H_{\mathrm{E}, \mathrm{L}}$, are adequately expressed using local normal vibrational coordinates which describe distortions of one $\left(\mathrm{MnF}_{6}\right)^{4-}$ octahedron (Höck et al 1983, Thomas 1987). We start by discussing the electron-lattice interaction.

In principle, the threefold orbital degenerate ${ }^{4} \mathrm{~T}_{\mathrm{gg}}$ state interacts with local modes of $a_{1}$, e and $t_{2}$ symmetry. However, the coupling with $t_{2}$ modes is quenched because of the strong Jahn-Teller effect (JTE) of the e modes, known as the Ham effect (Chen et al 1972). Restricting ourselves to linear terms we can express the contribution of a single manganese ion at site $n$ by

$$
H_{\mathrm{E}, \mathrm{L}}^{n}=A_{\mathrm{a}} \Lambda_{\mathrm{a}} Q_{\mathrm{a} ; n}+A_{\mathrm{e}} \Lambda_{\mathrm{e}, \Theta} Q_{\Theta ; n}+A_{\mathrm{e}} \Lambda_{\mathrm{e}, \varepsilon} Q_{\varepsilon ; n}
$$

where $\Lambda_{p}$ are the matrices

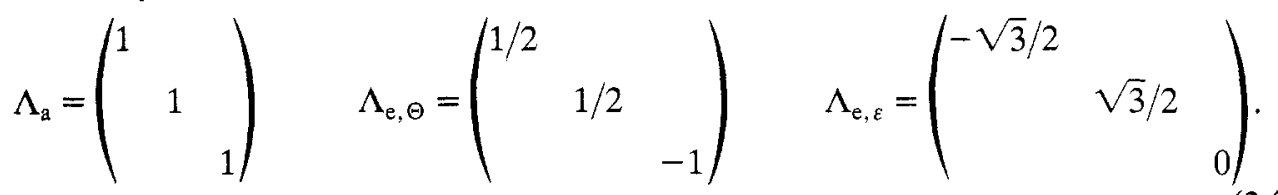

$Q_{\mathrm{a}}$ is the normal coordinate of the fully symmetric breathing mode of the $\mathrm{F}^{-}$and $\mathrm{Rb}^{+}$ ions around an excited $\mathrm{Mn}^{2+}$ ion. $Q_{\ominus}$ and $Q_{\varepsilon}$ describe modes of the tetragonal and rhombohedral distortion, respectively (figure 1). After optical excitation of the ${ }^{6} \mathrm{~A}_{1 \mathrm{~g}} \rightarrow{ }^{4} \mathrm{~T}_{1 \mathrm{~g}}$ transition, the forces $A_{\mathrm{a}}$ and $A_{\mathrm{e}}$ act on the $\mathrm{F}^{-}$ions and displace them to new equilibrium positions $Q_{\mathrm{a}, 0}, Q_{\ominus, 0}$ and $Q_{\varepsilon, 0}$. The distortion energy for the breathing mode of frequency $\omega_{\mathrm{a}}$ is

$$
E_{\mathrm{a}}=(M / 2) \omega_{\mathrm{a}}^{2} Q_{\mathrm{a}, 0}^{2} .
$$

$M$ is the mass of one $\mathrm{F}^{-}$ion. The symmetry-breaking e modes with frequency $\omega_{\mathrm{e}}$ determine the Jahn-Teller energy. Using the $z$ component of ${ }^{4} \mathrm{~T}_{1 \mathrm{~g}}$, for which the distortion $Q_{\varepsilon, 0}$ vanishes, we get a simple expression for the Jahn-Teller energy:

$$
E_{\Theta}=(M / 2) \omega_{\mathrm{e}}^{2} Q_{\Theta, 0}^{2} .
$$

Table 1 gives the coupling constants $A_{\mathrm{a}}$ and $A_{\mathrm{e}}$, the local mode frequencies $\hbar \omega_{\mathrm{a}}$ and $\hbar \omega_{\mathrm{e}}$, the displacement energies $E$, and the normal dispiacements $Q_{p, 0}$. All values have been derived from experimental data (Solomon and McClure 1972, 1974, Chen et al 1972).

We can now express the electron-lattice interaction of $n$ manganese ions, $H_{\mathrm{E}, \mathrm{L}}$, in second quantisation by

$$
H_{\mathrm{E}, \mathrm{L}}=\sum_{n} H_{\mathrm{E}, \mathrm{L}}^{n}=\sum_{n} \sum_{p}\left(A_{p} \Lambda_{p} Q_{p ; n}\right) c_{p n}^{+} c_{p n}
$$

where $p=\mathrm{a}, \Theta, \varepsilon$ and $A_{\Theta}=A_{\varepsilon}=A_{\mathrm{e}}$.

Finally, the lattice Hamiltonian $H_{\mathrm{L}}$ can be expressed by the same local vibrational 
Table 1. Electron-lattice coupling of the ${ }^{4} \mathrm{~T}_{1 \mathrm{~g}}$ state of $\mathrm{Mn}^{2+}$ in $\mathrm{RbMnF}_{3}$ : coupling constants $A$, local mode frequencies $\omega$, distortion energies $E$ and normal displacements $Q_{0}$ (Solomon and McClure 1972, 1974, Chen et al 1972).

\begin{tabular}{lllll}
\hline Mode & $A(\mathrm{~N})$ & $\hbar \omega\left(\mathrm{cm}^{-1}\right)$ & $E\left(\mathrm{~cm}^{-1}\right)$ & $Q_{0}(\AA)$ \\
\hline $\mathrm{a}$ & $-2.23 \times 10^{-9}$ & 400 & 703 & $Q_{a, 0}=-0.125$ \\
$\mathrm{e}$ & $+1.30 \times 10^{-9}$ & 287 & 458 & $Q_{\Theta .0}=-0.14^{\mathrm{a}}$ \\
& & & & $Q_{\varepsilon, 0}=0^{\mathrm{a}}$ \\
\hline
\end{tabular}

${ }^{\text {a }}$ Values are given for the $z$ component of ${ }^{4} \mathrm{~T}_{1 \mathrm{~g}}$

coordinates $Q_{p ; n}$ :

$$
\begin{aligned}
H_{\mathrm{L}}=\sum_{n} P_{\mathrm{a} ; n}^{2} / & 2 M+(M / 2) \omega_{\mathrm{a}}^{2} Q_{\mathrm{a} ; n}^{2}+\sum_{n}\left(P_{\Theta ; n}^{2}+P_{\varepsilon ; n}^{2}\right) / 2 M+(M / 2) \omega_{\mathrm{e}}^{2}\left(Q_{\Theta ; n}^{2}+Q_{\varepsilon ; n}^{2}\right) \\
& -\frac{1}{2} \sum_{n n^{\prime}} \sum_{p p^{\prime}} \nu_{n n^{\prime}}^{p p^{\prime}} Q_{p ; n} Q_{p^{\prime} ; n^{\prime}} .
\end{aligned}
$$

The coefficients $\nu_{n n^{\prime}}^{p p^{\prime}}$ determine the interaction between local vibrations and give rise to a dispersive optical phonon branch. We completely neglect this dispersion and drop the last term of equation (2.11).

After having explained the Hamiltonian of our system we shall now discuss the states which are relevant for the energy transfer between manganese ions. The electronic overlap $J$ will turn out to be of the order of several $\mathrm{cm}^{-1}$. The displacement energies $E_{\mathrm{a}}$ and $E_{\mathrm{e}}$, on the other hand, are some $100 \mathrm{~cm}^{-1}$, which means that the excitation is trapped by the local distortions. Transfer can only be accomplished by simultaneous tunnelling of electronic excitation and lattice distortion, i.e. by a Jahn-Teller polaron. In analogy to the exciton band (equation (2.5)) the polarons form a band with energies

$$
E(k)=E_{0}+2 J^{\prime} \cos (\boldsymbol{k} \cdot R)
$$

where $J^{\prime}$ describes the reduced bandwidth

$$
J^{\prime}=J \mathrm{e}^{-S} \text {. }
$$

In $\S 7$ we will show that the reduction factor $\mathrm{e}^{-s}$ is about 0.035 . An upper limit of $J^{\prime}$ may be estimated from a comparison with other manganese fluorides. For comparison, $\mathrm{MnF}_{2}$ has a JTE of magnitude similar to $\mathrm{RbMnF}_{3}$ but only half of the manganese nearestneighbour distance; Dietz et al (1970) estimate $J^{\prime} \leqslant 0.5 \mathrm{~cm}^{-1}$ for $\mathrm{MnF}_{2}$. This corresponds to a $\mathrm{Mn}-\mathrm{Mn}$ transfer time of $\tau \geqslant h / 2 J^{\prime} \simeq 10^{-10} \mathrm{~s}$ for a purely coherent transfer. We regard this time as a lower limit also for $\mathrm{RbMnF}_{3}$.

The coherence of the transfer will be quickly destroyed compared to the experimental timescale. The reason for this is an inhomogeneous broadening of the ${ }^{4} \mathrm{~T}_{1 \mathrm{~g}}$ polaron state which is larger than $J^{\prime}$. We measured in absorption a linewidth of about $1.2 \mathrm{~cm}^{-1}$. In $\mathrm{CsMnF}_{3}$, where two neighbouring $\mathrm{Mn}^{2+}$ ions have a distance $(a=5.8 \AA)$ nearly equal to $\mathrm{RbMnF}_{3}$, the coherence time was observed to be $10^{-7} \mathrm{~s}$ (Moncorge and Jacquier 1983). The experimental timescale, determined by the fastest decay of the ${ }^{4} \mathrm{~T}_{\mathrm{gg}}$ state, is about $30 \mu$ s (see $\$ 5$ ). For the time being we will therefore restrict our considerations of energy transfer to completely incoherent processes and discuss possible effects of coherence in $\S 6$.

So far we have discussed qualitatively the polaronic states of the manganese system. 
We now add some quantitative aspects by using the wavefunctions of the local Hamiltonian. Correctly speaking, we omit the transfer terms of the system Hamiltonian of equation (2.2), rewrite equation (2.2) as

$$
H=\sum_{n} H^{n}=\sum_{n} H_{\mathrm{E}}^{n}+H_{\mathrm{L}}^{n}+H_{\mathrm{E}, \mathrm{L}}^{n}
$$

and consider the eigenfunctions of $H^{n}$. For low temperatures the functions of the first excited state are simply Born-Oppenheimer products:

$$
\begin{aligned}
& \psi_{n}^{x}=\left|{ }^{4} \mathrm{~T}_{1 \mathrm{~g}}^{x}(n)\right\rangle\left|\chi_{n}^{x}\right\rangle=:\left|X_{n}\right\rangle\left|\chi_{n}^{x}\right\rangle \\
& \psi_{n}^{y}=\left|{ }^{4} \mathrm{~T}_{1 \mathrm{~g}}^{y}(n)\right\rangle\left|\chi_{n}^{y}\right\rangle=:\left|Y_{n}\right\rangle\left|\chi_{n}^{y}\right\rangle \\
& \psi_{n}^{z}=\left|{ }^{4} \mathrm{~T}_{\mathrm{lg}}^{z}(n)\right\rangle\left|\chi_{n}^{z}\right\rangle=:\left|Z_{n}\right\rangle\left|\chi_{n}^{z}\right\rangle .
\end{aligned}
$$

The states $|\chi\rangle$ belong to displaced harmonic oscillators:

$$
\begin{aligned}
& \chi^{x}(Q)=F_{\mathrm{a}}\left(Q_{\mathrm{a}}-Q_{\mathrm{a}, 0}\right) F_{\Theta}\left(Q_{\Theta}+Q_{\Theta, 0} / 2\right) F_{\varepsilon}\left(Q_{\varepsilon}-(\sqrt{3} / 2) Q_{\Theta, 0}\right) \\
& \chi^{y}(Q)=F_{\mathrm{a}}\left(Q_{\mathrm{a}}-Q_{\mathrm{a}, 0}\right) F_{\Theta}\left(Q_{\Theta}+Q_{\Theta, 0} / 2\right) F_{\varepsilon}\left(Q_{\varepsilon}+(\sqrt{3} / 2) Q_{\Theta, 0}\right) \\
& \chi^{z}(Q)=F_{\mathrm{a}}\left(Q_{\mathrm{a}}-Q_{\mathrm{a}, 0}\right) F_{\Theta}\left(Q_{\Theta}-Q_{\Theta, 0}\right) F_{\varepsilon}\left(Q_{\varepsilon}\right)
\end{aligned}
$$

where $F_{i}$ are one-dimensional oscillator functions in the vibrational ground state and $Q_{\mathrm{a}, 0}$ and $Q_{\Theta, 0}$ are the normal displacements as given in table 1. Although $\left|\chi^{\chi}\right\rangle,\left|\chi^{\nu}\right\rangle$ and $\left|\chi^{z}\right\rangle$ are not orthonormal their localisation is strong enough that the overlap can be neglected, i.e. $\left\langle\chi^{x} \mid \chi^{y}\right\rangle=\left\langle\chi^{y} \mid \chi^{z}\right\rangle=\left\langle\chi^{\chi} \mid \chi^{z}\right\rangle \simeq 0$. It is important to note that $\psi^{x}, \psi^{y}$ and $\psi^{z}$ are still degenerate. The Ham effect mentioned above leads to the reduction of nondiagonal electronic matrix elements of an arbitrary electronic operator $O$ (see table 1 ):

$$
\begin{aligned}
\left\langle\psi^{x}|O| \psi^{y}\right\rangle & =\langle X|O| Y\rangle\left\langle\chi^{x} \mid \chi^{y}\right\rangle \\
& =\langle X|O| Y\rangle \exp \left(-\frac{3}{2} E_{\mathrm{JT}} / \hbar \omega_{\mathrm{e}}\right)=\langle X|O| Y\rangle \times 0.09
\end{aligned}
$$

Similar to equation (2.15) the ground state $\psi_{n}^{0}$ of a $\mathrm{Mn}^{2+}$ ion may be described by the product of an electronic term and a vibronic term, but now the $\left\langle\chi_{n}^{0}\right\rangle$ belong to undisplaced harmonic oscillator functions:

$$
\psi_{n}^{0}=\left|{ }^{6} \mathrm{~A}_{1 \mathrm{~g}}(n)\right\rangle\left|\chi_{n}^{0}\right\rangle=:\left|A_{n}\right\rangle\left|\chi_{n}^{0}\right\rangle
$$

When uniaxial stress $\sigma$ is applied to the crystal the states are perturbed by a homogeneous strain field $\varepsilon$. The strain Hamiltonian may be written in analogy to $H_{\mathrm{E}, \mathrm{L}}$ (see equation (2.6))

$$
H_{\varepsilon}=\Sigma_{p} A_{p}^{\prime} \Lambda_{p} \varepsilon_{p}
$$

$\varepsilon_{p}$ are the irreducible components of the strain tensor and $\Lambda_{p}$ are the matrices of equation (2.7). Again the Ham effect suppresses the interaction with shear strain components so that we only consider $p=\mathrm{a}, \Theta, \varepsilon$. For the case of $\mathrm{RbMnF}_{3}$ we can assume that the constants $A_{p}^{\prime}$ of equation (2.19) are equal to the $A_{p}$ of equation (2.6) except for geometrical factors (Solomon and McClure 1974):

$$
A_{\mathrm{a}}^{\prime}=\sqrt{6} R_{0} A_{\mathrm{a}} \quad A_{\Theta}^{\prime}=A_{\varepsilon}^{\prime}=A_{\mathrm{e}}^{\prime}=(2 / \sqrt{ } 3) R_{0} A_{\mathrm{c}}
$$

where $R_{0}$ is the MnF bond length $R_{0}=2.125 \AA$.

Usually external stress is applied along the [ $\left[\begin{array}{ll}0 & 0\end{array}\right],\left[\begin{array}{lll}1 & 1 & 0\end{array}\right]$ and $\left[\begin{array}{lll}1 & 1 & 1\end{array}\right]$ axes. The strain 
components $\varepsilon_{\mathrm{a}}, \varepsilon_{\Theta}$ and $\varepsilon_{\varepsilon}$ for these directions are

[0 0101$]: \quad \varepsilon_{\mathrm{a}}=-\left(S_{11}+2 S_{12}\right) \sigma / 3$

[1 110$]: \quad \varepsilon_{\mathrm{a}}=-\left(S_{11}+2 S_{12}\right) \sigma / 3$

$$
\begin{aligned}
& \varepsilon_{\Theta}=2\left(S_{11}-S_{12}\right) \sigma / 3 \quad \varepsilon_{\varepsilon}=0 \\
& \text { [11 1 ] : } \varepsilon_{\mathrm{a}}=-\left(S_{11}+2 S_{12}\right) \sigma / 3 \quad \varepsilon_{\Theta}=0 \quad \varepsilon_{\varepsilon}=0 \text {. }
\end{aligned}
$$

The symmetric strain $\varepsilon_{\mathrm{a}}$ causes a shift of the exciton band and the tetragonal strain $\varepsilon_{\Theta}$ causes a splitting. We have determined the energy shift to be

$$
\mathrm{d} E / \mathrm{d} \sigma_{\mathrm{a}}=-95 \mathrm{~cm}^{-1} \mathrm{GPa}^{-1}
$$

independent of the stress direction. The splitting with [ $\left[\begin{array}{lll}0 & 0 & 1\end{array}\right]$ stress as shown in figure 2 is

$$
\mathrm{d} E / \mathrm{d} \sigma_{\Theta}=285 \mathrm{~cm}^{-1} \mathrm{GPa}^{-1} .
$$

Here the lower level is the twofold orbital degenerate $\left(\psi^{x}, \psi^{y}\right)$ state, the upper level is the orbital singlet state $\psi^{z}$. With [ $\left[\begin{array}{ll}1 & 1\end{array}\right]$ stress half of the splitting is achieved in accordance with equation (2.21), and the $\psi^{z}$ state is the lowest. Application of [1 111$]$ stress gives only a shift but no splitting as expected for a $\mathrm{T} \times$ e Jahn-Teller system.

So far we have neglected spin-orbit coupling and the antiferromagnetic exchange field. We will discuss these effects in $\S 6$.

It is necessary to emphasise that in the initial state of luminescence each orbital state $\psi^{i}$ is connected with a stable axial distortion along one of the three [0 01$]$ axes. The stress splitting is usually larger than the thermal energy $k_{\mathrm{B}} T$ if the experiments are done at liquid helium temperature (see figure 2). Therefore by applying uniaxial stress a certain orbital state is selected as well as the corresponding distortion. In $\S 4$ we describe energy transfer experiments under uniaxial stress. It is expected that the transfer rate depends on the orbital state selected. When, for example, [ $\left[\begin{array}{lll}1 & 1 & 0\end{array}\right]$ stress is applied, the transfer occurs exclusively via the $\psi^{z}$ state. Under $\left[\begin{array}{lll}0 & 0 & 1\end{array}\right]$ stress, however, transfer is accomplished by the degenerate states $\psi^{x}$ and $\psi^{y}$ which are not orthonormal. Since the lifetime is long, we expect that there are transitions between these states.

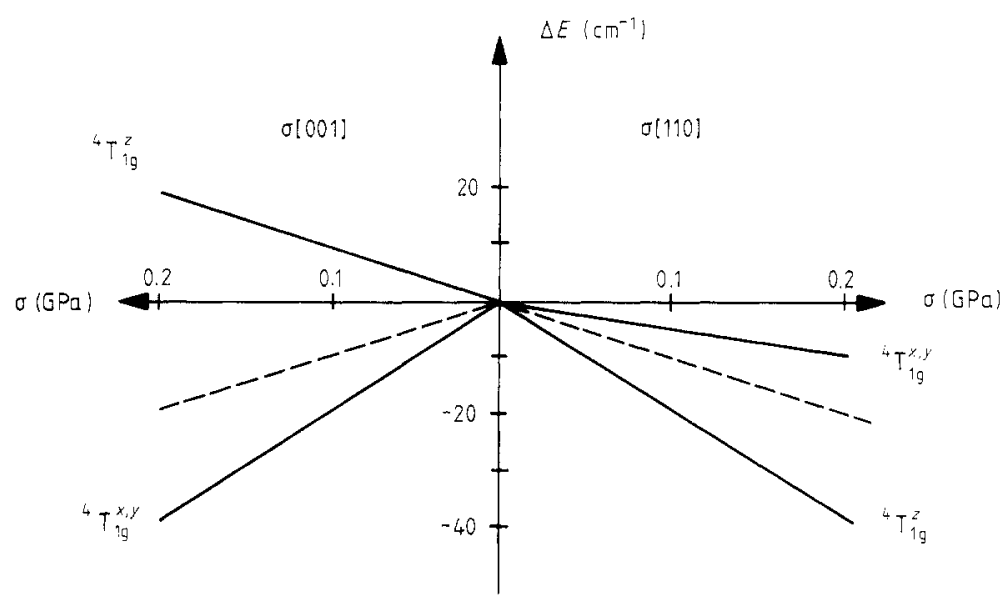

Figure 2. Splitting pattern of the manganese ${ }^{4} T_{18}$ state in $\mathrm{RbMnF}_{3}$ by uniaxial stress. Exchange

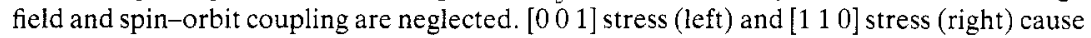
a shift (broken lines) and a splitting. 


\section{Energy transfer}

\subsection{Rate equations}

The experiments described below are concerned with energy transfer of ${ }^{4} \mathrm{~T}_{1 \mathrm{~g}}$ exciton polarons. We show in $\S 5$ that the measured lifetime is mainly determined by transfer to traps. Two kinds of traps, called $\mathrm{G}$ and $\mathrm{R}$ traps, are observable (figure 3 ). The depths of the traps measured relative to the intrinsic ${ }^{4} \mathrm{~T}_{1 \mathrm{~g}}$ exciton level are $27 \mathrm{~cm}^{-1}$ for a $\mathrm{G}$ trap and $123 \mathrm{~cm}^{-1}$ for an $\mathrm{R}$ trap. These energies remain constant even if uniaxial stress is applied. We did not observe energy transfer between these traps. The trap luminescence decays with a rate constant of $\lambda=16 \mathrm{~s}^{-1}$; we consider this as the radiative decay rate of the traps. Although the detailed structure of these traps in $\mathrm{RbMnF}_{2}$ is not well known we use the model developed for $\mathrm{MnF}_{2}$ where trap emission from doped crystals has been analysed (Greene et al 1968). It was concluded that divalent impurities, such as $\mathrm{Ca}, \mathrm{Mg}$ or $\mathrm{Zn}$, occupy manganese sites. Those are always present in concentrations of some ppm. The ${ }^{4} \mathrm{~T}_{1 \mathrm{~g}}$ energy of nearest- and next-nearest-neighbour $\mathrm{Mn}^{2+}$ ions is slightly reduced by the impurities' strain field. Hence these $\mathrm{Mn}^{2+}$ ions act as efficient shallow traps of ${ }^{4} \mathrm{~T}_{1 \mathrm{~g}}$ excitons. The radiative decay rate of the intrinsic exciton will be the same as or even smaller than the rate $\lambda=16 \mathrm{~s}^{-1}$ of the traps. Transfer rates, however, are orders of magnitude larger ( $\mathrm{see} \S 5$ ). We therefore may consider $\lambda$ as the radiative decay rate of the intrinsic exciton $(\mathrm{E})$ as well.

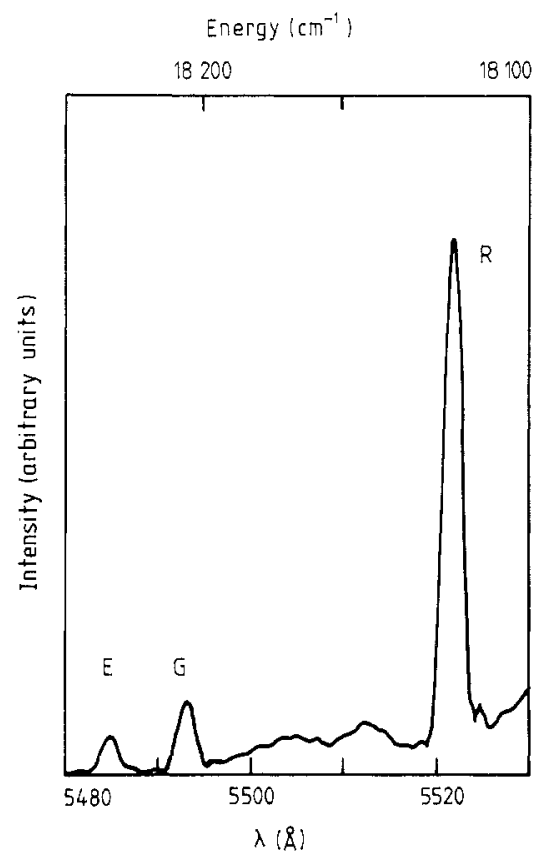

Figure 3. Emission spectra of $\mathrm{RbMnF}_{3}$ near the $\mathrm{Mn}^{2+}{ }^{4} \mathrm{~T}_{1 \mathrm{~g}}$ exciton $\mathrm{E}$ (zero-phonon, zero-magnon transition). $T=1.4 \mathrm{~K}$, no stress applied, excitation by a $10 \mathrm{~ns}$ laser pulse at $\lambda=5530 \AA$, gate time $25 \mu \mathrm{s}$. G, R: emission from local traps.

A phenomenological description of the excitonic energy transfer to the $G$ and $R$ traps is obtained by using rate equations with averaged energy transfer rates $k_{\mathrm{G}}$ and $k_{\mathrm{R}}$ :

$$
\begin{aligned}
& \mathrm{d} n_{\mathrm{E}} / \mathrm{d} t=-\left(\lambda+k_{\mathrm{G}}+k_{\mathrm{R}}\right) n_{\mathrm{E}} \\
& \mathrm{d} n_{\mathrm{G}} / \mathrm{d} t=-\lambda n_{\mathrm{G}}+k_{\mathrm{G}} n_{\mathrm{E}} \\
& \mathrm{d} n_{\mathrm{R}} / \mathrm{d} t=-\lambda n_{\mathrm{R}}+k_{\mathrm{R}} n_{\mathrm{E}} .
\end{aligned}
$$


Assuming $\delta(t)$ pulse excitation the solutions for the occupation numbers of the intrinsic exciton states $n_{\mathrm{E}}$ and for the trap states $n_{\mathrm{G}}$ and $n_{\mathrm{R}}$ are

$n_{\mathrm{E}}(t)=n_{\mathrm{E}}(t=0) \exp (-\mu t)$

$n_{\mathrm{G}}(t)=\left[n_{\mathrm{G}}(t=0)+n_{\mathrm{E}}(t=0) k_{\mathrm{G}} / K\right] \exp (-\lambda t)-n_{\mathrm{E}}(t=0) k_{\mathrm{G}} / K \exp (-\mu t)$

$n_{\mathrm{R}}(t)=\left[n_{\mathrm{R}}(t=0)+n_{\mathrm{E}}(t=0) k_{\mathrm{R}} / K\right] \exp (-\lambda t)-n_{\mathrm{E}}(t=0) k_{\mathrm{R}} / K \exp (-\mu t)$

with $K:=k_{\mathrm{G}}+k_{\mathrm{R}}$ and $\mu:=\lambda+K$. The emission intensities are simply given by

$$
I_{\mathrm{E}, \mathrm{R}, \mathrm{G}}(t)=n_{\mathrm{E}, \mathrm{R}, \mathrm{G}}(t) \lambda \text {. }
$$

\subsection{The random-walk model}

The simple model given above describes a strictly exponential decay of the exciton with a rate $\mu=\lambda+K$ which is in general not observed. Instead one has to use a more general form for the decay process

$$
I(t) \propto \exp (-\lambda t) \Phi(t) .
$$

It was the purpose of a series of sophisticated calculations to determine the transfer function $\Phi(t)$ (see e.g. Huber 1987). In the present study we use a simple randomwalk model which describes a nearest-neighbour hopping transfer. Effects of coherent transfer are neglected. Equation (3.4) takes the form

$$
I\left(t_{n}\right) \propto \exp \left(-\lambda n \tau_{\mathrm{H}}\right) \Phi_{n}
$$

where the continuous time coordinate $t$ is replaced by

$$
t_{n}=n \tau_{\mathrm{H}} \quad \text { and } \quad \Phi_{n}=\Phi\left(t_{n}\right)
$$

with $n$ the number of steps and $\tau_{\mathrm{H}}$ the nearest-neighbour transfer time. We consider an excitation which migrates in one of the two FCC spin sublattices. The intrinsic manganese ions are assumed to be statistically substituted by traps. If a trap site is reached by the excitation the transfer is stopped. Then $\Phi_{n}$ is the probability that the excitation has not reached a trap after $n$ steps, i.e. the survival probability.

Under uniaxial stress the transfer is expected to become anisotropic. The properties of anisotropic energy transfer can be described well by the random-walk model. For this purpose we consider the local transfer rate for one step, starting with a ${ }^{4} \mathrm{~T}_{1 \mathrm{~g}}$ excitation which is located at the centre of a cube (figure 4). If the excitation is in the $\psi^{z}$ state we can distinguish the following processes by symmetry:

(i) transfer to the four nearest neighbours in the $x y$ plane with a rate $T_{x y}$, and

(ii) transfer to the eight nearest neighbours in the $x z$ and $y z$ planes with a rate $T_{z}$.

Although the lattice symmetry is cubic, the local rates $T_{x y}$ and $T_{z}$ for the orbital singlet $\psi^{z}$ can differ substantially. An analogous consideration holds if we start with a $\psi^{x}$ or $\psi^{y}$ state. However, when two or even three orbital components are degenerate the excitation will not stay in its initial substate. In our model we assume that during each transfer step a scattering from the actual substate into one of the same energy can occur. For the stress-free case this leads to an isotropic transfer on a three-dimensional FCC lattice with $T_{x y}=T_{z}$.

It should be possible to observe a characteristic change of the transfer function $\Phi(t)$ when stress is applied and the anisotropy $T_{x y} / T_{z}$ becomes large enough. The magnitude of this anisotropy is not known a priori. In principle we have to distinguish between two 


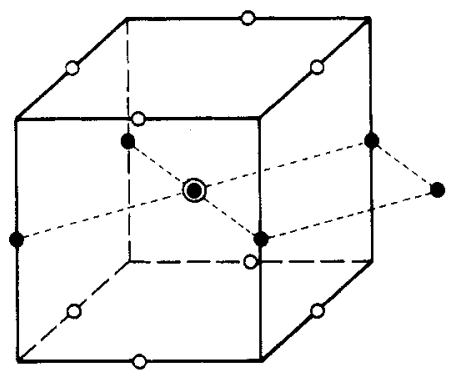

(a)

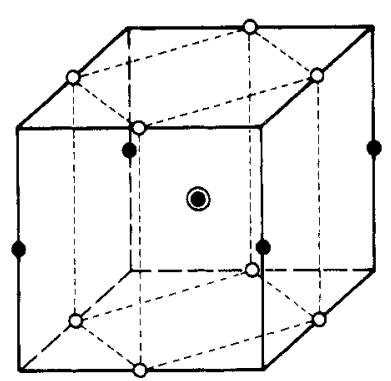

(b)

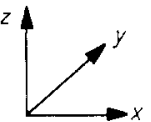

Figure 4. FCC structure built up by manganese ions of one magnetic sublattice (central ion and 12 neighbours). Ions marked by and $\bigcirc$, respectively, are equivalent considering the ${ }^{4} \mathrm{~T}_{\mathrm{Ig}}^{z}$ substate. (a) Simple quadratic lattice formed by equivalent manganese ions of one $x y$ plane. $(b)$ Bcc structure consisting of the central ion and its eight equivalent neighbours.

situations for the non-degenerate state $\psi_{z}: T_{x y}>T_{z}$ or $T_{x y}<T_{z}$. Ultimately, either $T_{z}$ or $T_{x y}$ may be zero.

In the first situation, we have a large anisotropy: [ 110$]$ stress selects the $\psi^{z}$ state for the transfer. Assuming a vanishing $T_{z}$ the transfer for [ $\left[\begin{array}{lll}1 & 1 & 0\end{array}\right]$ stress will be confined to planes perpendicular to the $z$ axis; these planes form simple quadratic lattices. For $\left[\begin{array}{lll}0 & 0 & 1\end{array}\right]$ stress we expect transfer on a three-dimensional BCC lattice because the selected substates $\psi_{x}$ and $\psi_{y}$ are degenerate.

In the second situation, we consider the limit $T_{x y}=0$ for the $\psi_{z}$ state. For [ 1110$]$ stress we obtain transfer on a three-dimensional BCC lattice, and for [ $\left[\begin{array}{lll}0 & 0\end{array}\right]$ stress a walk on a three-dimensional FCC lattice. The difference in $\Phi_{n}$ for a walk on a FCC or on a BCC lattice is actually very small (see e.g. Blumen and Zumofen 1981). This is in contrast to our experimental results which show a drastic difference between the decay under [ $\left[\begin{array}{lll}0 & 0 & 1\end{array}\right]$ stress and [ $\left.\begin{array}{lll}1 & 1 & 0\end{array}\right]$ stress ( further and assume $T_{x y} \geqslant T_{z}$.

The dependence of $\Phi_{n}$ on the trap concentration $p$ for a two- and three-dimensional lattice is plotted in figure 5, as broken curves (Blumen and Zumofen 1981, Zumofen and Blumen 1982). In three dimensions $\Phi_{n}$ decays nearly exponentially and, with fixed ratio $\tau_{\mathrm{H}} / p$, is practically independent of $p$. However, for a two-dimensional lattice, $\Phi_{n}$ shows a non-exponential behaviour at least for short times. Moreover, $\Phi_{n}$ depends strongly on the trap concentration $p$. We must emphasise that in our transfer experiments we do not really have a two-dimensional system; instead we observe a two-dimensional walk in single planes of a three-dimensional lattice. In order to compare the data of Blumen and Zumofen with our experiments we assume that the trap concentration in each plane varies according to a binomial distribution. Then we find that the decay of $\Phi_{n}$ remains non-exponential even at very long times (full curves in figure 5).

In order to estimate the magnitude of transfer anisotropy, calculations of $\Phi_{n}$ between the limiting cases of purely two- and purely three-dimensional transfer are necessary. This was achieved by computer simulations. We constructed a model FCC lattice with $40^{3}$ points which corresponds to one sublattice of manganese sites with equal spin orientation. For each random-walk process the lattice was randomly occupied with $p=1 \%$ traps; one lattice point was randomly chosen as starting point. At each step of 


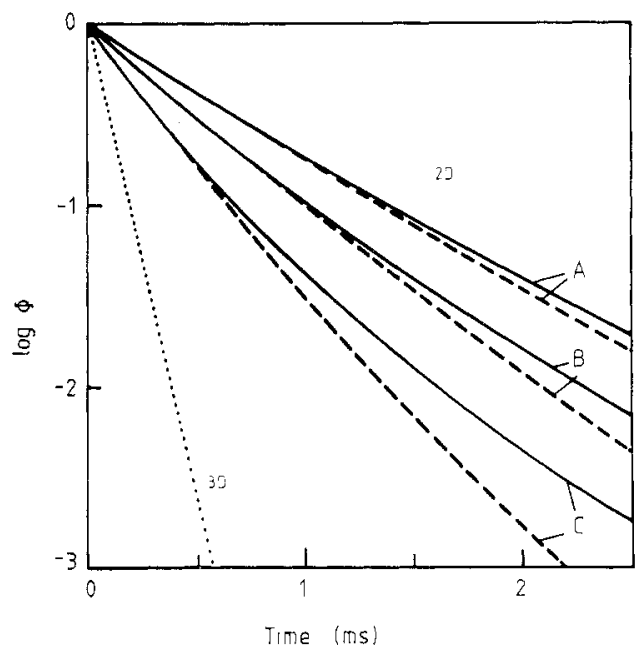

Figure 5. Survival rate $\Phi_{n}$ of the random walk described in $\$ 3.2$-the ratio of hopping time $\tau_{\mathrm{H}}$ and trap concentration $p$ for all curves is kept constant $\left(\tau_{\mathrm{H}} / p=10^{-4} \mathrm{~s}\right):(\cdots)$ 3D BCC lattice (Blumen and Zumofen 1981); $\Phi_{n}$ is independent of $p$. (-- ) 2D square lattice (Zumofen and Blumen 1982); $\Phi_{n}$ depends on $p: \mathrm{A}, p=1 \mathrm{ppm}\left(\tau_{\mathrm{H}}=\right.$ $\left.10^{-10} \mathrm{~s}\right) ; \mathrm{B}, p=100 \mathrm{ppm}\left(\tau_{\mathrm{H}}=10^{-8} \mathrm{~s}\right) ; \mathrm{C}, p=1 \%$ $\left(\tau_{\mathrm{H}}=10^{-6} \mathrm{~s}\right)$. ( $(-)$ Same as broken curves but the trap concentration in one plane of the crystal varies according to a binomial distribution.

the process the excitation jumps to one of the neighbouring lattice points until, after $i$ steps, a trap is reached. The whole process was repeated $10^{4}$ times. Then the survival rate $\Phi_{n}$ was computed using

$$
\Phi_{n}=\left(10^{4}-\sum_{i=1}^{n} N_{i}\right) / 10^{4}
$$

where $N_{i}$ is the number of excitations which reach a trap after $i$ transfer steps. By varying the ratio $T_{x y} / T_{z}$ of the local transfer rates, the transfer for any given anisotropy can be simulated. For example, we can reproduce the results of Blumen and Zumofen for a three-dimensional walk. For $T_{z}=0$ (two-dimensional transfer) our results differ from theirs (broken curve for $1 \%$ in figure 5). However, our simulated $\Phi_{n}$ coincides with the full curve in figure 5 where the distribution of the trap concentration is included. The results of our simulations for $1<T_{x y} / T_{z}<\infty$ are shown in figure 12 .

\section{Experimental details}

The two samples of $\mathrm{RbMnF}_{3}$ studied here were cut along a [ $\left[\begin{array}{lll}0 & 0 & 1\end{array}\right]$ and a [ $\left[\begin{array}{lll}1 & 1 & 0\end{array}\right]$ plane and were prepared from the same Bridgman-grown single crystal to guarantee equal impurity concentrations. The samples measured $1.3 \times 1.3 \times 1.6 \mathrm{~mm}$ and were cooled in a combined bath/gas-flow cryostat. The temperature was measured and controlled by a calibrated carbon-glass resistor. Below $4.2 \mathrm{~K}$ the accuracy of the temperature was better than $0.05 \mathrm{~K}$.

The uniaxial stress was applied to the sample in the cryostat by a lever system which was activated by an external force. The system was constructed so that any torsion at the sample was avoided. The stress was measured by a strain gauge at the lever system and additionally controlled by the exciton splitting.

The luminescence was excited by radiation of a dye laser (Lambda Physik, type FL2002) operated with Coumarin 153. The dye laser was pumped by an excimer laser (Lambda Physik, type EMG 102). The dye laser pulses had a duration of $10 \mathrm{~ns}$, an energy of $10 \mathrm{~mJ}$ and a repetition rate of $3 \mathrm{~Hz}$. The slow repetition rate allowed for a nearly 
complete decay of long-lived luminescence states and prevented heating of the crystal. The laser beam was expanded to a diameter of $2 \mathrm{~mm}$ to avoid non-linear effects during excitation.

The luminescence was recorded with a $1 \mathrm{~m}$ grating monochromator (Spex 1704). The detection system consisted of a cooled photomultiplier (Hamamatsu R1463) which was electrically gated inactive during the laser pulse. The signals were fed into a photon counting system and the amplified pulses were stored time-selected in a multi-channel analyser.

In order to prepare a proper initial state, resonant excitation into the ${ }^{4} \mathrm{~T}_{1 \mathrm{~g}}$ exciton should be used. Because of the small transition probability of the zero-phonon transition ${ }^{4} \mathrm{~T}_{1 \mathrm{~g}} \rightarrow{ }^{6} \mathrm{~A}_{1 \mathrm{~g}}$ the luminescence yield is much higher when excited via phonon-sideband absorption. We therefore used this non-resonant excitation at $\lambda=553 \mathrm{~nm}$ after we had verified that neither the luminescence shape nor the dynamics were affected.

\section{Results}

\subsection{Low-temperature measurements: $T<4 \mathrm{~K}$}

The exciton luminescence decays exponentially if no external stress is applied. The decay rate $\mu$ is the sum of the radiative rate $\lambda$ and the overall transfer rate $K$ (equation (3.2)). We measured $\mu=20840 \mathrm{~s}^{-1}$ at $1.4 \mathrm{~K}$. As shown in figure 6 the decay is slower when [ $\left.\begin{array}{lll}0 & 0 & 1\end{array}\right]$ stress is applied but is still exponential. However, if [ $\left[\begin{array}{lll}1 & 1 & 0\end{array}\right]$ stress is applied the slowing down is not only much more pronounced but the decay also becomes nonexponential. For the radiative rate $\lambda$, which is determined by the decay of traps (see $\S 3.1$ ), we obtain $\lambda=16 \mathrm{~s}^{-1}$ with and without stress. We therefore interpret the change of the excitonic emission dynamics under stress as a change of the transfer rate $K$.

A corresponding effect is observed in the rise of the trap luminescence. The traps are filled after excitation of the intrinsic exciton levels, which is observed as a rise of the trap luminescence. This is shown for the $\mathrm{R}$ traps in figure 7. Although a qualitative explanation of the three curves in analogy to figure 6 is easy, the quantitative evaluation has to take into account the stress splitting of the $R$ levels which is $48 \mathrm{~cm}^{-1} \mathrm{GPa}^{-1}$ for [ $\left.\begin{array}{lll}1 & 1 & 0\end{array}\right]$ stress. Furthermore luminescence of the $G$ traps is influenced by saturation effects. We therefore restrict the following considerations to the dynamics of the intrinsic exciton luminescence.

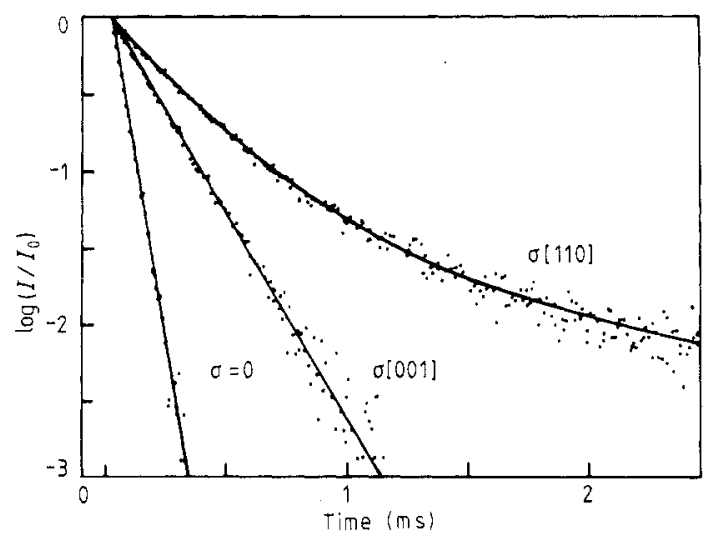

Figure 6. Emission dynamics of the intrinsic ${ }^{4} \mathrm{~T}_{1 \mathrm{~g}}$ exciton of $\mathrm{RbMnF}_{3}$ without and with applied stress of $0.165 \mathrm{GPa} . \quad T=1.4 \mathrm{~K}$, excitation with a 10 ns laser pulse via phononsideband absorption $(\lambda=553 \mathrm{~nm})$. 


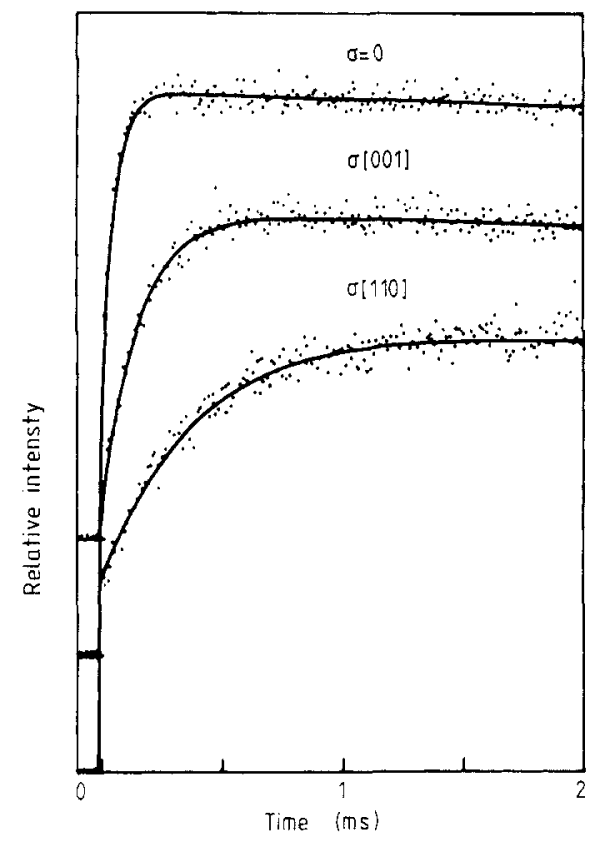

Figure 7. Time-resolved emission from trap R; experimental conditions are the same as in figure 6 .

If the decay is exponential the decay rate $\mu$ gives the transfer rate. Here we neglect $\lambda$ which is always small compared with $K$. For non-exponential decay we can calculate an average decay time

$$
\langle\tau\rangle=\int_{0}^{\infty} t I(t) \mathrm{d} t / \int_{0}^{\infty} I(t) \mathrm{d} t .
$$

Again neglecting the radiative decay we obtain the transfer rate $K$ from

$$
K=\langle\tau\rangle^{-1} \text {. }
$$

We have analysed $K$ as a function of the stress $\sigma$. In figure 8 ([ $\left[\begin{array}{lll}0 & 0 & 1\end{array}\right]$ stress) and figure 9

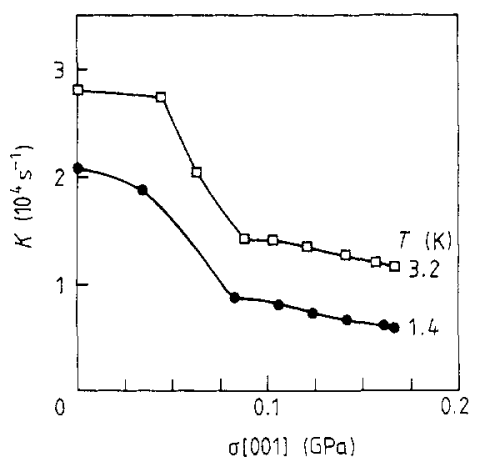

Figure 8. Transfer rate $K$ as a function of $\left[\begin{array}{lll}0 & 0 & 1\end{array}\right]$ stress at 1.4 and $3.2 \mathrm{~K}$. Data taken from the exponential exciton emission.

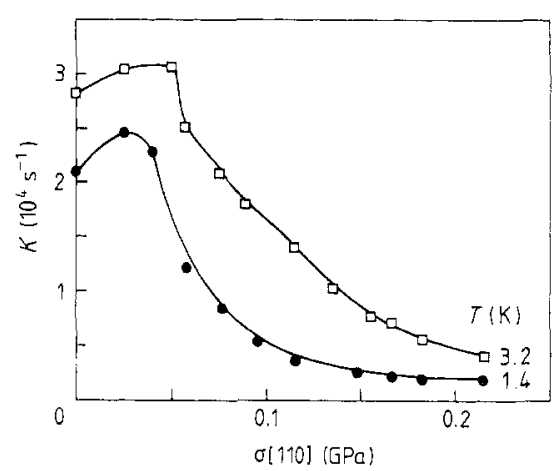

Figure 9. Transfer rate $K$ as a function of [ $\left[\begin{array}{lll}1 & 1 & 0\end{array}\right]$ stress at 1.4 and $3.2 \mathrm{~K}$. Data taken from the exciton emission. For $T=1.4 \mathrm{~K}$ and $\sigma \geqslant 0.08 \mathrm{GPa}$, when the decay is not exponential, we used equations (5.1) and (5.2). 
([1 110$]$ stress) we show the results for two temperatures, 1.4 and $3.2 \mathrm{~K}$. The stress dependences $K(\sigma)$ for the two stress directions are complicated and are affected differently by temperature. Moreover, the decay of the exciton luminescence is exponential for temperatures above $2.2 \mathrm{~K}$ even for $\left[\begin{array}{lll}1 & 1 & 0\end{array}\right]$ stress. At low stress values, $K$ is practically constant. There is an intermediate range where $K$ decreases. At high stress values $K$ is again nearly constant or shows a weak decrease with stress. In $\S 6$ we will show that in the weak stress region the excitonic states are mixed states due to the spin-orbit interaction whereas at high stress they are purely orbital states $\psi^{x}, \psi^{y}$ and $\psi^{z}$. Only under the latter conditions may we assume that the transfer occurs between $\left(\psi^{x}, \psi^{y}\right)$ states for $\left[\begin{array}{lll}0 & 0 & 1\end{array}\right]$ stress and between $\psi^{z}$ states for [1 110$]$ stress.

\subsection{The temperature range $4 K \leqslant T \leqslant 8.5 K$}

In this range the $\mathrm{G}$ traps, which have a depth of $27 \mathrm{~cm}^{-1}$, are emptied into the exciton band by thermal activation. The decay of the $G$ trap emission can be described by a temperature-dependent decay rate

$$
\lambda(T)=\lambda(T=0)+B \exp \left(-\Delta E / k_{\mathrm{B}} T\right)
$$

where $\lambda(T=0)=16 \mathrm{~cm}^{-1}, \Delta E=37 \pm 3 \mathrm{~cm}^{-1}$ and $B \simeq 10^{7} \mathrm{~s}^{-1}$. No stress dependence of $\Delta E$ or $B$ is observed.

\subsection{The temperature range $8.5 \mathrm{~K} \leqslant T \leqslant 12 \mathrm{~K}$}

A rapid decrease of the intrinsic exciton lifetime with increasing temperature is observed. The dependence can be fitted assuming an activation process

$$
\mu(T)=\mu(T=8.5 \mathrm{~K})+B \exp \left(-\Delta E / k_{\mathrm{B}} T\right)
$$

with $\Delta E=40 \pm 5 \mathrm{~cm}^{-1}$ and $B \simeq 3 \times 10^{7} \mathrm{~s}^{-1}$, independent of stress. The risetime of the $\mathrm{R}$ trap emission is also reduced. Its temperature dependence can be described with identical parameters. On the other hand its intensity and its decay time do not vary with temperature for $8.5 \mathrm{~K} \leqslant T \leqslant 12 \mathrm{~K}$. Therefore the observations indicate a higher activation level which makes rapid transfer possible. It is unlikely that this level belongs to the intrinsic ${ }^{4} \mathrm{~T}_{1 \mathrm{~g}}$ level scheme since the activation energy is independent of the stress splitting of this state. Similar activation processes have been observed in $\mathrm{KMnF}_{3}$ (Riederer 1975) and $\mathrm{MnF}_{2}$ (Wilson et al 1979) but have not been satisfactorily explained.

\subsection{Temperatures above $12 \mathrm{~K}$}

Above $12 \mathrm{~K}$ thermal depletion of the $\mathrm{R}$ traps is observed which is also described by equation (5.3) using $\Delta E=150 \pm 15 \mathrm{~cm}^{-1}$ and $B \approx 3 \times 10^{10} \mathrm{~s}^{-1}$. Again the thermal activation is stress-independent. $\Delta E$ exceeds the trap depth $\left(123 \mathrm{~cm}^{-1}\right)$ by $27 \pm 15 \mathrm{~cm}^{-1}$. This may be due to the fact that $R$ traps are directly emptied into the higher level $40 \mathrm{~cm}^{-1}$ above the intrinsic exciton level (see above).

\section{Discussion}

\subsection{Transfer rates and level structure}

We have seen in $\S 5$ that the measured decay rate of the intrinsic exciton luminescence is at least two orders of magnitude larger than the radiative decay rate. This was explained 
by energy transfer to traps. Two kinds of traps, the $G$ and $R$ traps, can be directly observed by their luminescence. When uniaxial stress is applied a slowing down of the exciton luminescence decay and of the rise of trap luminescence is observed. This may have two reasons:

(i) the transfer rate from a $\mathrm{Mn}^{2+}$ ion to a neighbouring trap is reduced, or

(ii) the transfer rate between intrinsic $\mathrm{Mn}^{2+}$ ions is reduced.

There are two observations which are not consistent with the first possibility. First, the trap depth is found to be independent of stress. Secondly, the parameter $B$ in equation (5.3), which may give an estimate of the local transfer rate from a neighbouring $\mathrm{Mn}^{2+}$ ion to a $\mathrm{G}$ trap, is $B=10^{7} \mathrm{~s}^{-1}$, independent of stress. This is three orders of magnitude higher than a typical exciton transfer rate $K=10^{4} \mathrm{~s}^{-1} \cdot K^{-1}$ therefore describes the average diffusion time of an excitation to a trap, and a reduction of $K$ must be caused by weaker transfer between intrinsic manganese ions. We have therefore excluded the first possibility and shall use the second process as a basis for the following interpretation.

Next we want to discuss the correlation between the transfer rate and the level structure of the ${ }^{4} \mathrm{~T}_{1 \mathrm{~g}}$ state. Three orbital components combined with spin $\frac{3}{2}$ lead to an overall degeneracy of 12 which is lifted by the antiferromagnetic exchange field, by spinorbit coupling and by external stress. We have diagonalised the complete $12 \times 12$ matrix of the ${ }^{4} \mathrm{~T}_{1 \mathrm{~g}}$ substates given by Solomon (1972) and determined energies and eigenvectors as a function of stress. We obtained the following results (figure 10).
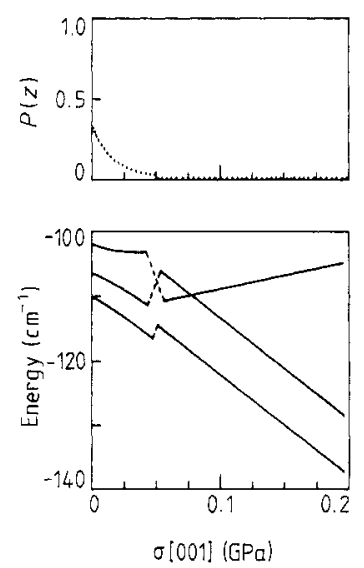
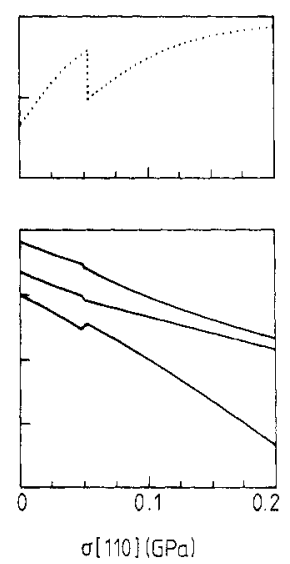

Figure 10. Fine structure of $\mathrm{Mn}^{2+}{ }^{4} \mathrm{~T}_{1 \mathrm{~g}}$ in $\mathrm{RbMnF}_{3}$. Results from diagonalisation of the complete perturbation matrix. Left side, $\left[\begin{array}{lll}0 & 0 & 1]\end{array}\right]$ stress; right side, $\left[\begin{array}{lll}1 & 1 & 0\end{array}\right]$ stress. Remember that at $\sigma=$ $0.05 \mathrm{GPa}$ the spins are oriented parallel to the stress field. Lower curves: energy of the lowest three sublevels relative to the unperturbated state. Upper curves: percentage of the $z$ orbital within the lowest substate $P(z)$.

The exchange field splits the ${ }^{4} \mathrm{~T}_{1 \mathrm{~g}}$ state into four spin components separated by $83 \mathrm{~cm}^{-1}$. While in the stress-free case the spins are parallel to a [1 111 ] direction, they align parallel to the stress axis when stress of more than $0.05 \mathrm{GPa}$ is applied (Eastman 1967). Spin-orbit coupling and stress do not mix the four spin components but only cause a splitting of each component into three sublevels. In order to interpret the experiments it is sufficient to consider only the lowest spin component since-in absorption-only these three sublevels of ${ }^{4} \mathrm{~T}_{1 \mathrm{~g}}$ are observed. The correlation between spin-orbit coupling and stress leads to a splitting pattern as shown in figure 10 (lower part). For $\sigma=0$ we see that the three sublevels are within $8 \mathrm{~cm}^{-1}$. With increasing stress the pattern becomes similar to that of figure 2 . We have carried out absorption experiments and could verify the computed splitting patterns very well.

Luminescence in $\mathrm{RbMnF}_{3}$ at liquid helium temperature can only be seen from the 
lowest excited substate. Therefore we assume that an excited upper sublevel relaxes into the lowest before transfer occurs. A description of energy transfer using the model of $\S 3$ is only possible if we meet the condition of pure orbital eigen-states. In the upper curves of figure 10 the calculated probability of the system to be in the $\psi^{z}$ state is plotted versus stress. Without stress the system is in states determined by the spin-orbit coupling, and the probability of finding the system in one of the orbital states $\psi^{i}$ is $\frac{1}{3}$. For high $\left[\begin{array}{lll}1 & 1 & 0\end{array}\right]$ stress the lowest level is the singlet state $\psi^{z}$. A [ 0001$]$ stress of more than $0.05 \mathrm{GPa}$ leaves a doublet structure with components separated by about $10 \mathrm{~cm}^{-1}$ and consisting only of $\psi^{x}$ and $\psi^{y}$. Our calculations show that both these levels consist of $\psi^{x}$ and $\psi^{y}$ with equal probability.

From a comparison of figures 8,9 and 10 we conclude that the reduction of the transfer rate with stress is clearly related to demixing of spin-orbit states. The experimental results from figure $8\left(\begin{array}{lll}0 & 0 & 1\end{array}\right]$ stress) show that a strong decrease of the transfer rate is observed until the stress has reached about $0.08 \mathrm{GPa}$. Then the transfer rate stays practically constant. Here the lowest sublevel consists of the orbital doublet $\left(\psi^{x}, \psi^{y}\right)$. The increase of the transfer rate with increasing temperature between 1.4 and $3.2 \mathrm{~K}$ is independent of stress and therefore cannot be explained by thermally induced transfer to higher sublevels.

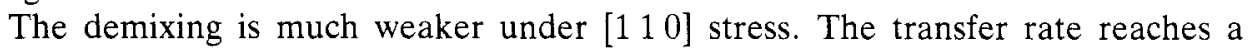
constant low value at a stress of about $0.18 \mathrm{GPa}$, in agreement with the slow development of a pure orbital $z$ state (figure 10). Raising the temperature will allow thermal activation into the $\left(\psi^{x}, \psi^{y}\right)$ levels. The mixing of the substates by temperature and demixing by [1 110$]$ stress are competing processes as is evident from figure 9. In the following discussions we will assume that a sufficiently high uniaxial stress is applied. This will leave us with a pure orbital doublet state $\left(\psi^{x}, \psi^{y}\right)$ for [0 011$]$ stress and a pure orbital singlet state $\psi^{z}$ for $\left[\begin{array}{lll}1 & 1 & 0\end{array}\right]$ stress experiments.

\subsection{Energy transfer as a random-walk process}

As we have discussed in $\S 2$ the dominating transfer mechanism is incoherent. We therefore discuss now incoherent energy transfer between manganese ions. This requires that after each transfer step the phase coherence between $\mathrm{Mn}^{2+}$ ions is destroyed. It is at present not possible to decide whether this assumption is completely fulfilled. We will further restrict the transfer to nearest-neighbour $\mathrm{Mn}^{2+}$ ions placed on one magnetic FCC sublattice. Then we can apply the random-walk model described in $\$ 3.2$. The walk is characterised by a hopping time $\tau_{\mathrm{H}}$.

As the exact trap concentration $p$ is not known we tested $p=1 \mathrm{ppm}, 100 \mathrm{ppm}$ and an unrealistically high concentration of $1 \%$. In figure 11 (full curves) the results of the random-walk model for two-dimensional transfer are plotted as in figure 5 (full curves). In order to plot the computed decay curves versus time, a specific transfer time $\tau_{\mathrm{H}}$ must be chosen. We have determined $\tau_{\mathrm{H}}$ by fitting the experimental decay curves observed under sufficiently high $\left[\begin{array}{lll}1 & 1 & 0\end{array}\right]$ stress. We used the curves taken at $\sigma=0.213 \mathrm{GPa}$ and $T=1.4 \mathrm{~K}$, where the orbital substates of ${ }^{4} \mathrm{~T}_{1 \mathrm{~g}}$ are completely demixed. The experimental points are also plotted in figure 11. With the hopping time $\tau_{\mathrm{H}}$ found in this way and a fixed trap concentration $p$ we computed a luminescence decay for the three-dimensional transfer via doublet states $\psi^{x}$ and $\psi^{y}$ (broken curves in figure 11). A comparison with experimental decay curves obtained with $\sigma[001] \geqslant 0.08 \mathrm{GPa}$ (when the demixing of spin-orbitals by [ $\left[\begin{array}{lll}0 & 0 & 1\end{array}\right]$ stress is complete) show that both decay processes are well reproduced over one-and-a-half orders of magnitude of intensity. Table 2 summarises the 

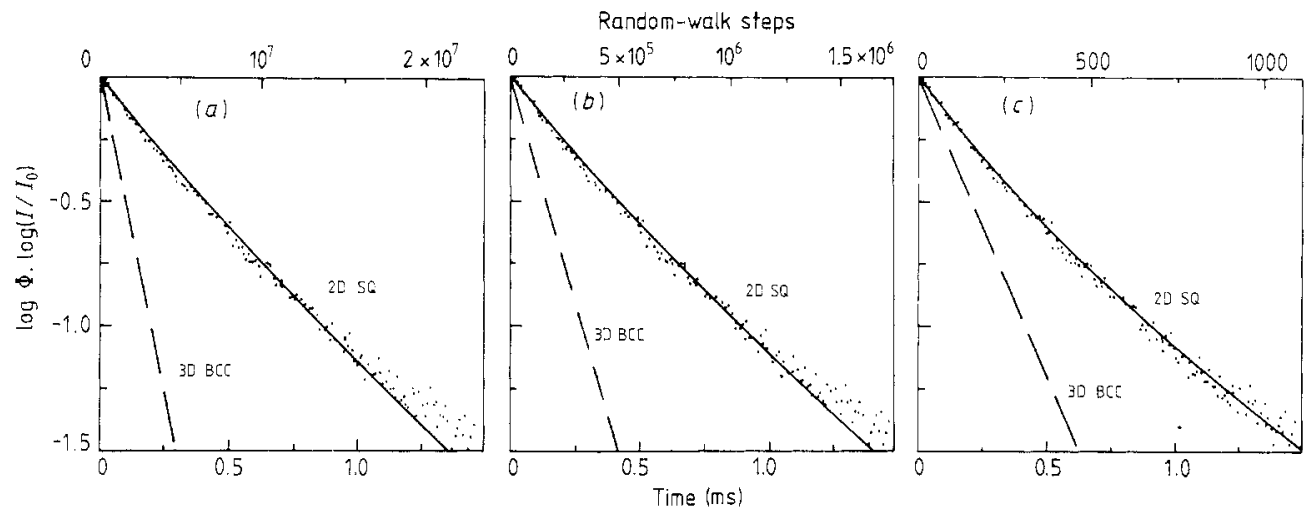

Figure 11. Comparison of the results of the random-walk model with experiments under [1 10 ] stress: (points) experimental exciton decay at high stress $(\sigma=0.21 \mathrm{GPa})$ and low temperature $(1.4 \mathrm{~K}) ;(-)$ best fit of the $2 \mathrm{D}$ random walk (see figure 5 , full curves) to the experiments. Trap concentration arbitrarily chosen: $(a) p=1 \mathrm{ppm},(b) p=100 \mathrm{ppm},(c)$ $p=1 \%$. Hopping time $\tau_{\mathrm{H}}$ derived from the fit $(a) 6.4 \times 10^{-11} \mathrm{~s},(b) 8.6 \times 10^{-9} \mathrm{~s},(c)$ $1.35 \times 10^{-6} \mathrm{~s}$. (----) Survival rate $\Phi_{n}$ for $3 \mathrm{D}$ random walk using the same $p$ and $\tau_{\mathrm{H}}$.

relevant data and parameters obtained from the random-walk model and luminescence experiments. For a realistic trap concentration of $p=100 \mathrm{ppm}$ the transfer rate becomes $\tau^{-1}=8339 \mathrm{~s}^{-1}$. This agrees well with the experimental value $K=8500 \mathrm{~s}^{-1}$ obtained at $\sigma\left[\begin{array}{lll}1 & 1 & 0\end{array}\right]=0.08 \mathrm{GPa}$.

However, a careful analysis shows that after long times the observed luminescence decays slower than calculated. Using the unrealistic high trap concentration of $p=1 \%$ a better agreement can be obtained. There may be two reasons for this behaviour:

(i) Random fields in the crystal form regions of exciton confinement and may inhibit the random walk of the excitation.

Table 2. Transfer rates $K$ under [001] stress. (a) Some experimental transfer rates $K$ measured under [0 01 l] stress; remember that for $\sigma[001] \geqslant 0.08 \mathrm{GPa}$ transfer occurs in the $\psi^{x}$ and $\psi^{y}$ substate. (b) Transfer rates $K$ for [ $\left[\begin{array}{lll}0 & 1\end{array}\right]$ stress predicted by the three-dimensional random-walk model for some values of trap concentration $p$; the hopping time $\tau_{\mathrm{H}}$ was taken from a best fit of a two-dimensional random walk to [ $\left[\begin{array}{ll}1 & 1\end{array}\right]$ stress experiments (see figure 11).

\begin{tabular}{llr}
\hline$(a)$ & & \\
$T(\mathrm{~K})$ & $\sigma(\mathrm{GPa})$ & $K\left(\mathrm{~s}^{-1}\right)$ \\
\hline 1.4 & 0.08 & 8500 \\
1.4 & 0.20 & 4600 \\
3.2 & 0.08 & 14000 \\
3.2 & 0.20 & 10100 \\
\hline$(b)$ & & \\
$p$ & $\tau_{\mathrm{H}}(\mathrm{s})$ & $K\left(\mathrm{~s}^{-1}\right)$ \\
\hline $10^{-6}$ & $0.64 \times 10^{-10}$ & 11189 \\
$10^{-5}$ & $0.75 \times 10^{-9}$ & 9615 \\
$10^{-4}$ & $0.86 \times 10^{-8}$ & 8339 \\
$10^{-3}$ & $1.07 \times 10^{-7}$ & 7029 \\
$10^{-2}$ & $1.35 \times 10^{-6}$ & 5435 \\
\hline
\end{tabular}




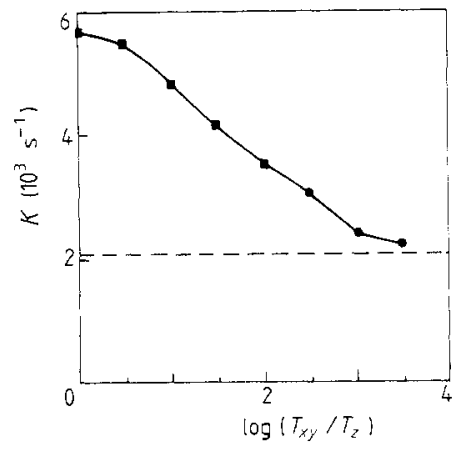

Figure 12. Computer simulations of energy transfer as a function of local transfer anisotropy $T_{x y} / T_{z}$. Model FCC lattice with $40^{3}$ points, trap concentration $p=1 \%$. The transfer rate $K$ was derived directly from the exponential $\Phi_{n}$ $\left(T_{x y} / T_{z} \leqslant 100\right)$ or according to equations (5.1) and (5.2). The broken horizontal line is the limiting value of the transfer rate for $T_{z}=0$.

(ii) A coherent transfer could be possible for a time $\tau_{\mathrm{C}}>\tau_{\mathrm{H}}$. Then $\tau_{\mathrm{C}}$ rather than $\tau_{\mathrm{H}}$ is the ruling time for incoherent transfer. When the average decay time $\langle\tau\rangle$ is fixed a longer $\tau_{\mathrm{C}}$ corresponds to higher trap concentrations.

Which of the two reasons hold for the transfer process cannot be decided from our data.

So far we have only considered pure two- and three-dimensional transfer. Our computer simulations have yielded transfer rates and decay behaviour at arbitrary anisotropy. For the general case of non-exponential decay we can calculate an average lifetime $\langle\tau\rangle$ and a transfer rate $K=\langle\tau\rangle^{-1}$ from the simulation according to equation (5.1). The result is plotted in figure 12 as a function of the anisotropy $T_{x y} / T_{z}$. It is interesting to note that there is an approximately logarithmic decrease of $K$ with $T_{x y} / T_{z}$. A variation of $T_{x y} / T_{z}$ between 1 and 10 changes the transfer rate $K$ only by $15 \%$. A drastic change of the transfer properties is only seen when $T_{x y} / T_{z}$ increases by more than two orders of magnitude. Then the transfer rate is reduced by nearly a factor of 3 and, furthermore, non-exponential decay is observed. This was also seen in the luminescence decay experiments when sufficiently high [ $\left[\begin{array}{lll}1 & 1 & 0\end{array}\right]$ stress was applied. We therefore conclude that the computer simulations support our model of two-dimensional energy transfer induced by $\left[\begin{array}{lll}1 & 1 & 0\end{array}\right]$ stress.

\section{A microscopic model for the transfer}

In this section we give an estimate for the transfer matrix element $J_{n n^{\prime}}^{\prime}$ (see equations (2.4), (2.5) and (2.12)) for the singlet $\psi^{z}$ state between nearest-neighbour $\mathrm{Mn}^{2+}$ ions with parallel spin moments. In the context of transfer anisotropy we want to clarify how $J_{n n^{\prime}}^{\prime}$ differs for non-equivalent neighbours $n^{\prime}$ (see figure 4). We use the product of electronic and vibronic wavefunctions for the ground state and for the excited state (see equations (2.15) and (2.18)) at the lattice point $R_{n}$ :

$$
\psi_{n}^{0}=\left|A_{n}\right\rangle\left|\chi_{n}^{0}\right\rangle \quad \psi_{n}^{z}=\left|Z_{n}\right\rangle\left|\chi_{n}^{z}\right\rangle
$$

with the electronic states $A={ }^{6} \mathrm{~A}_{1 \mathrm{~g}}$ and $Z={ }^{4} \mathrm{~T}_{\mathrm{1g}}^{z}$. The equilibrium positions of the local vibrational modes described by $\chi$ correspond to the relaxed environment when the central $\mathrm{Mn}^{2+}$ ion at $R_{n}$ is in the electronic $|A\rangle$ or $|Z\rangle$ state, respectively. The transfer matrix element is then given by

$$
J_{n n^{\prime}}^{\prime}=\left\langle\psi_{n}^{z} \psi_{n^{\prime}}^{0}|J| \psi_{n}^{0} \psi_{n^{\prime}}^{z}\right\rangle
$$

The operator $J$ describing the Coulomb interaction between $3 \mathrm{~d}^{5}$ electrons at different 
manganese sites is

$$
J=\sum_{j=1}^{5} \sum_{k=6}^{10} e^{2} /\left|r_{j}-r_{k}\right| .
$$

Here $j=1, \ldots, 5$ enumerates the electrons at $R_{n}$ and $k=6, \ldots, 10$ those at $R_{n^{\prime}} . J$ acts only on the electronic part of the wavefunction. The transfer matrix element can be separated into an electronic part $J_{n n^{\prime}}$ and a vibronic part

$$
J_{n n^{\prime}}^{\prime}=J_{n n^{\prime}} \cdot\left\langle\chi_{n}^{z} \chi_{n^{\prime}}^{0} \mid \chi_{n}^{0} \chi_{n^{\prime}}^{z}\right\rangle
$$

with

$$
J_{n n^{\prime}}=\left\langle Z_{n} A_{n^{\prime}}|J| A_{n} Z_{n^{\prime}}\right\rangle .
$$

We neglect a direct overlap of the vibrational wavefunctions at two different lattice sites. This seems to be reasonable considering the distances $R_{\mathrm{Mn}-\mathrm{F}}=2.15 \AA$ and $R_{\mathrm{Mn} \uparrow-\mathrm{Mn} \uparrow}=6.0 \AA$ and a decay of local distortions proportional to $R^{-3}$. Then the vibronic part exhibits no anisotropy because we have

$$
\left\langle\chi_{n}^{z} \chi_{n^{\prime}}^{0} \mid \chi_{n}^{0} \chi_{n^{\prime}}^{z}\right\rangle=\left\langle\chi_{n}^{z} \mid \chi_{n}^{0}\right\rangle \cdot\left\langle\chi_{n^{\prime}}^{0} \mid \chi_{n^{\prime}}^{z}\right\rangle
$$

and we can make use of the translational symmetry

$$
\left\langle\chi_{n}^{z} \mid \chi_{n}^{0}\right\rangle=\left\langle\chi_{n^{\prime}}^{0} \mid \chi_{n^{\prime}}^{z}\right\rangle
$$

Inserting harmonic oscillator functions, the vibronic part of the matrix element is given by

$$
\left|\left\langle\chi_{n}^{z} \mid \chi_{n}^{0}\right\rangle\right|^{2}=\mathrm{e}^{-S}=0.035
$$

with the Huang-Rhys factor $S$ calculated with the data from table 1

$$
S=\sum_{p=\mathrm{a}, \Theta, \varepsilon}\left(M \omega_{p} / 2 \hbar\right) Q_{p, 0}^{2}
$$

Here we see how the polaronic motion reduces the transfer matrix element. The electronic part of the matrix element $J_{n n^{\prime}}$ has the form

$$
\begin{aligned}
J_{n n^{\prime}}=\int Z_{n}^{*}( & \left.r_{1}, \ldots, r_{5}\right) A_{n^{\prime}}^{*}\left(r_{6}, \ldots, r_{10}\right) \\
& \quad \times\left(\sum_{j, k} e^{2} / r_{j k}\right) A_{n}\left(r_{1}, \ldots, r_{5}\right) Z_{n^{\prime}}\left(r_{6}, \ldots, r_{10}\right) \mathrm{d} \tau_{1} \ldots \mathrm{d} \tau_{10} .
\end{aligned}
$$

The multi-electron wavefunctions can be expressed as products of one-electron states which are base vectors of the irreducible representation of the cubic point group $\mathrm{O}_{\mathrm{h}}$ (Solomon and McClure 1974). The ground state is simply given by

$$
|A\rangle=\left|\mathrm{t}^{3} \mathrm{e}^{2}\right\rangle=-\left|\xi^{+} \eta^{+} \xi^{+} \Theta^{+} \varepsilon^{+}\right\rangle .
$$

The ${ }^{4} \mathrm{~T}_{1 \mathrm{~g}}$ state is approximately

$$
\left.{ }^{4} \mathrm{~T}_{1 \mathrm{~g}}\right\rangle \simeq\left|\mathrm{t}^{4} \mathrm{e}^{1}\right\rangle .
$$

Therefore the $z$ state can be written as

$$
|Z\rangle \simeq\left|\mathbf{t}^{4} \mathrm{e}^{1}, \boldsymbol{Z}\right\rangle=-\left|\xi^{+} \eta^{+} \xi^{+} \xi^{-} \Theta^{+}\right\rangle .
$$


A two-ion state like $\left|A_{n} Z_{n^{\prime}}\right\rangle$ can be derived from a common ground state $\left|A_{n} A_{n^{\prime}}\right\rangle$ by oneelectron creation $\left(c^{+}\right)$and annihilation $(c)$ operators

$$
\left|A_{n} Z_{n^{\prime}}\right\rangle=c\left(\varepsilon^{+}, n^{\prime}\right) c^{+}\left(\zeta^{-}, n^{\prime}\right)\left|A_{n} A_{n^{\prime}}\right\rangle
$$

In order to calculate the matrix element of $J$ we write the operator $J$ as

$$
J=\frac{1}{2} \sum_{i j k l=1}^{20}\langle i j|J| k l\rangle c_{j}^{+} c_{i}^{+} c_{k} c_{l}
$$

where $i, j, k, l$ enumerate all possible one-electron states at $R_{n}$ and $R_{n^{\prime}}$. If the overlap of single electron orbitals at different sites is neglected the matrix elements of $J$ consist only of two-electron Coulomb and exchange integrals of the form

$$
\begin{aligned}
J_{n n^{\prime}}=: C- & E \\
= & \int \mathrm{d} \tau_{1} \mathrm{~d} \tau_{2}\left\{\varepsilon^{+}\left(r_{1}-R\right) \cdot\left[\zeta^{-}\left(r_{1}-R\right)\right]^{*}\right\}^{*}\left(e^{2} / r_{12}\right)\left\{\varepsilon^{+}\left(r_{2}\right) \cdot\left[\zeta^{-}\left(r_{2}\right)\right]^{*}\right\} \\
& -\int \mathrm{d} \tau_{1} \mathrm{~d} \tau_{2}\left\{\varepsilon^{+}\left(r_{1}-R\right) \cdot\left[\varepsilon^{+}\left(r_{1}\right)\right]^{*}\right\}^{*}\left(e^{2} / r_{12}\right) \\
& \times\left\{\zeta^{-}\left(r_{2}-R\right) \cdot\left[\zeta^{-}\left(r_{2}-R\right)\right]^{*}\right\}
\end{aligned}
$$

with $R=R_{n}-R_{n^{\prime}} . \varepsilon$ and $\zeta$ are real functions given by

$$
\varepsilon=\frac{1}{2}\left[\left(x^{2}-y^{2}\right) / r^{2}\right] f(r) \quad \zeta=\left(x y / r^{2}\right) f(r) .
$$

We use a Slater function to approximate the radial dependence of $\varepsilon$ and $\zeta$ (Griffith 1971)

$$
f(r) \propto r^{2} \mathrm{e}^{-r / a_{0}}
$$

with $a_{0}=0.28 \AA$. Using equations (7.16) and (7.17) the radial dependence of the exchange term becomes $\exp \left(-2 R / a_{0}\right)$. This is a rapidly decreasing function of $R$. At $R=6 \AA$ this term has practically vanished.

We have calculated the relative values of the Coulomb term of equation (7.15) for non-equivalent neighbours $R_{\mathrm{n}^{\prime}}$. This term describes the interaction between two clouds of electronic charges at a distance of $6 \AA$, each consisting of one electron in the $\varepsilon$ state and one in the $\zeta$ state. The two clouds have a very small overlap. Therefore they may be approximated by spheres of radius $2.75 \AA$ and we can perform a numerical integration of the Coulomb term. Then we obtain the following result: the Coulomb term, connecting nearest neighbours in the $x y$ plane, is larger by a factor of 17.6 than the term connecting nearest neighbours with different $z$ coordinates. The transfer rates contain the square of the Coulomb matrix elements, and therefore we find

$$
T_{x y} / T_{z} \simeq 310 \text {. }
$$

This result supports very well the suggestion of anisotropic transfer and shows that the transfer in the singlet state is practically two-dimensional.

\section{Summary and conclusions}

The effect of vibronic coupling on the energy transfer of ${ }^{4} \mathrm{~T}_{1 \mathrm{~g}}$ excitons in $\mathrm{RbMnF}_{3}$ was investigated. The electronic $\mathrm{Mn}^{2+}$ state couples with local a and e modes of the $\mathrm{MnF}_{6}$ 
octahedra. The $\mathrm{T} \times \mathrm{e}$ Jahn-Teller effect leads to a static distortion which can be oriented by uniaxial stress.

The exciton luminescence was investigated by time-resolved spectroscopy. Its dynamics were determined by energy transfer to shallow traps. The traps were used as probes for the exciton transfer. The transfer rate was found to be reduced when uniaxial stress was applied. The most drastic effect was observed with [ $\left[\begin{array}{lll}1 & 1 & 0\end{array}\right]$ stress which, below $T=2.5 \mathrm{~K}$, induced a very slow non-exponential decay.

The general effect of uniaxial stress is a demixing of ${ }^{4} \mathrm{~T}_{1 \mathrm{~g}}$ orbitals. We have calculated this demixing by diagonalising the $12 \times 12$ perturbation matrix of ${ }^{4} \mathrm{~T}_{1 \mathrm{~g}}$ taking into account spin-orbit coupling, exchange field and stress. The analysis shows that sufficiently strong $\left[\begin{array}{lll}1 & 1 & 0\end{array}\right]$ stress selects a pure ${ }^{4} \mathrm{~T}_{\mathrm{1g}}^{z}$ state as the initial state for the luminescence.

Completely incoherent transfer was considered using a random-walk model. Computer simulations were performed to study the transfer for arbitrary anisotropy. Only if the transfer is completely confined to lattice planes can the experiments with

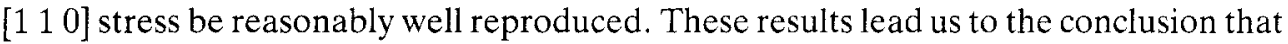
transfer under sufficiently strong $\left[\begin{array}{lll}1 & 1 & 0\end{array}\right]$ stress is completely two-dimensional, whereas under $\left[\begin{array}{lll}0 & 0 & 1\end{array}\right]$ stress (when ${ }^{4} \mathrm{~T}_{1 \mathrm{~g}}^{x}$ and ${ }^{4} \mathrm{~T}_{1 \mathrm{~g}}^{\mathrm{g}}$ are the initial states) the transfer is isotropic.

In order to perform a microscopic analysis of the transfer rates we have calculated the transfer integrals for the ${ }^{4} \mathrm{~T}_{1 \mathrm{~g}}^{z}$ state which are determined by Coulomb integrals using Slater functions. In fact we obtained an in-plane transfer rate which is 300 times larger than the off-plane rate. This strongly supports the proposed model of energy transfer.

The study of transport mechanisms in systems with strong electron-lattice coupling becomes increasingly interesting. We have shown in this paper that for the cubic perovskite $\mathrm{RbMnF}_{3}$ the excitonic energy transfer is modified by the following polaronic effects: enhancement of localisation, reduction of coherence, and possibility of demixing of the electronic substates by uniaxial stress.

\section{References}

Blumen A and Zumofen G 1981 J. Chem. Phys. 75892

Chen M Y, McClure D S and Solomon E I 1972 Phys, Rev. B 61690

Dietz R E, Meixner A E, Guggenheim H J and Misetich A 1970 J. Lumin. 1/2279

Eastman D E 1967 Phys. Rev. 156645

Greene R L, Sell D D, Feigelson R S, Imbusch G F and Guggenheim H J 1968 Phys. Rev. 171600

Griffith J S 1971 The Theory of Transition-Metal Ions (Cambridge: CUP)

Höck K-H, Nickisch H and Thomas H 1983 Helv. Phys. Acta 56237

Huber D L 1987 Transfer and trapping of optical excitation, in Excited-State Spectroscopy ed. U M Grassano and N Terzi (Amsterdam: North-Holland)

Moncorge $\mathrm{R}$ and Jacquier $\mathrm{B} 1983$ Optical dynamics in concentrated $\mathrm{Mn}(2+)$ systems, in Collective Excitations in Solids ed. B Di Bartolo (New York: Plenum)

Pacheco D P 1983 Collective excitations in concentrated $\mathrm{Mn}(2+)$ systems, in Collective Excitations in Solids, ed. B Di Bartolo (New York: Plenum)

Riederer H 1975 Diploma Thesis Regensburg

Solomon E I 1972 PhD Thesis Princeton

Solomon E I and McClure D S 1972 Phys. Rev. B 61697 1974 Phys. Rev. B 94690

Strauss E, Gerhardt V and Riederer H 1976 J. Lumin. 12/13239

Thomas H 1987 Phonons and electron-phonon interaction at localized centres, in Excited-State Spectroscopy, ed. U M Grassano and N Terzi (Amsterdam: North-Holland)

Wilson B A, Yen W M, Hegarty J and Imbusch G F 1979 Phys. Rev. B 194238

Zumofen G and Blumen A 1982 J. Chem. Phys. 763713 